\title{
On Application of Radiation Loss Factor in the Prediction of Sound Transmission Loss of a Honeycomb Panel
}

\author{
Leping Feng and Sathish Kumar R. \\ The Marcus Wallenberg Laboratory for Sound and Vibration Research, KTH (The Royal Institute of Technology), \\ SE-100 44 Stockholm, Sweden
}

(Submitted 25 February 2010, resubmitted 10 June 2011, accepted 8 December 2011)

\begin{abstract}
The application of the radiation loss factor in the prediction of sound transmission loss of a lightweight, orthotropic sandwich panel is investigated in this paper. Comparisons with measurements show that predictions often underestimate the sound transmission loss of the panel around the corresponding critical frequency when the measured loss factor, which in principle includes the radiation loss factor, is used. This is due to the measurement methods used for the loss factor and the band average. It is thus recommended to use the loss factor measured at low frequencies plus the theoretical radiation loss factor in order to improve the prediction of the sound transmission loss of a honeycomb panel around the critical frequency.
\end{abstract}

\section{INTRODUCTION}

The radiation loss factor of a structure is usually defined as the ratio of the sound energy radiated per radian to the reversible vibratory energy of the structure. ${ }^{1}$ For a plate-like structure, this is in direct proportion to the radiation efficiency of the structure and the characteristic impedance of the surrounding media, and in inverse proportion to the surface density of the panel and the frequency concerned. As a result, the radiation loss factor is important for structural vibration in air only when the panel is light and when the frequency concerned is not too high. When the sound transmission problem of a panel is investigated, the radiation loss factor is often neglected, unless the material loss factor is very low and unless the properties around the critical frequency of the panel are concerned. If the loss factor of a structure is obtained from the measurements made in air, the radiation loss is considered to have been included.

A honeycomb panel is a type of lightweight, sandwich panel with a honeycomb core of hexagon cells. It is often approximated as an orthotropic panel when the frequency is below the first dilatational resonance of the panel. The effective mechanical properties of the panel are commonly used in the prediction model. Because of the frequency-dependent property of the bending stiffness, the critical frequency of a honeycomb panel is spread in a frequency interval instead of a single frequency. ${ }^{2,3}$ This property makes the sound insulation property of a honeycomb panel much worse than that of a solid, isotropic one with the same surface density. Damping may improve the sound insulation properties of the panel from around the corresponding critical frequency and above. ${ }^{4}$

Due to the anisotropic properties, the sound transmission loss of a honeycomb panel cannot be predicted as a nor- mal single-leaf by using the mass law, even if the equivalent, frequency-dependent bending stiffness is applied. Various commercial software, such as AutoSEA, NOVA (analytical sandwich model) and VA1 (periodic structures module), are available to predict the sound transmission loss of a sandwich panel, including honeycomb panels when the detail of the panel is available. However, although the prediction for other frequency regions is rather good, the results obtained around the critical frequency often show much lower sound transmission loss than measurements, even when a measured loss factor is used and even when a very high structural loss factor is introduced in calculation. ${ }^{5}$ The purpose of this paper is to investigate the reasons for the discrepancy and the way to make a better prediction around the critical frequency area.

\section{HONEYCOMB PANELS TESTED}

Two honeycomb panels were investigated. They were of the same thickness and same facings but with different cell sizes: Panel 1 had a cell size of $6.4 \mathrm{~mm}$ and panel 2 had a cell size of $19.2 \mathrm{~mm}$. The $6.4 \mathrm{~mm}$ cell size is commonly found in practical cases, while the $19.2 \mathrm{~mm}$ cell size is not commonly used for industry. The reason to choose such a large cell size is to see the difference in acoustical properties between a normal honeycomb panel and a "badly designed" honeycomb panel. Both facings and core were made of aluminum. As described in reference, ${ }^{3}$ the shape of the honeycomb cells of a typical aluminum core is often rather irregular, which may produce some extra errors in the prediction of the sound transmission loss. The basic parameters of the honeycomb panels tested are listed in Table 1, where the loss factors are averages of measurements in $1 / 3$ octave bands with a central frequency from $100 \mathrm{~Hz}$ up to $1000 \mathrm{~Hz}$. The size of the panels under test was $1.25 \times 2.05$ meters. 